\title{
Realms: A Foundation for Spatial Data Types in Database Systems ${ }^{1}$
}

\author{
Ralf Hartmut Güting \\ Markus Schneider \\ Praktische Informatik IV, FernUniversität Hagen \\ Postfach 940, D-5800 Hagen, Germany \\ gueting@fernuni-hagen.de,schneide@fernuni-hagen.de
}

\begin{abstract}
Spatial data types or algebras for database systems should (i) be fully general (which means, closed under set operations, hence e.g. a region value can be a set of polygons with holes), (ii) have formally defined semantics, (iii) be defined in terms of finite representations available in computers, (iv) offer facilities to enforce geometric consistency of related spatial objects, and (v) be independent of a particular DBMS data model, but cooperate with any. We offer such a definition in two papers. The central idea, introduced in this (first) paper, is to use realms as geometric domains underlying spatial data types. A realm as a general database concept is a finite, dynamic, user-defined structure underlying one or more system data types. A geometric realm defined here is a planar graph over a finite resolution grid. Problems of numerical robustness and topological correctness are solved below and within the realm layer so that spatial algebras defined above a realm enjoy very nice algebraic properties. Realms also interact with a DBMS to enforce geometric consistency on object creation or update.
\end{abstract}

Keywords: Spatial data types, algebra, realm, finite resolution, numerical robustness, topological correctness, geometric consistency.

1 This work was supported by the DFG (Deutsche Forschungsgemeinschaft) under grant Gu 293/1-1. 


\section{Introduction}

We consider a spatial database system to be a full-fledged DBMS with additional capabilities for the representation and manipulation of geometric data. As such, it provides the database technology needed to support applications such as geographic information systems. The standard DBMS view for the organization of spatial information is the following: A database consists of several classes of objects. A spatial object is just an object with an associated value ("attribute") of a spatial data type, such as, for example, POINT, LINE, or REGION. This is true regardless of whether the DBMS uses a relational, complex object, object-oriented or some other data model. Hence the definition and implementation of spatial data types is probably the most fundamental issue in the development of spatial database systems.

Although spatial data types (SDTs) are used routinely in the description of spatial query languages (e.g. [LiN87, JoC88, SvH91]), have been implemented in some prototype systems (e.g. [RoFS88, OrM88, Gü89]), and some formal definitions have been given [Gü88a, ScV89, GaNT91], there is still no completely satisfactory solution available according to the following criteria:

- Generality. The geometric objects used as SDT values should be as general as possible. For example, a region value should be able to represent a collection of disjoint areas each of which may have holes. More precisely, this means that the domains of data types POINT, LINE, and REGION must be closed under union, intersection, and difference of their underlying point sets. This allows for the definition of powerful data type operations with nice closure properties.

- Rigorous definition. The semantics of SDTs, that is, the possible values for the types and the functions associated with the operations, must be defined formally to avoid ambiguities for the user and the implementor.

- Finite resolution. The formal definitions must take into account the finite representations available in computers. This has so far been neglected in definitions of SDTs. It is left to the programmer to close this gap between theory and practice which leads rather inevitably not only to numerical but also topological errors.

- Treatment of geometric consistency. Distinct spatial objects may be related through geometric consistency constraints (e.g. adjacent regions have a common boundary). The definition of SDTs must offer facilities to enforce such consistency.

- General object model interface. Spatial data types as such are rather useless; they need to be integrated into a DBMS data model and query language. However, a definition of SDTs should be valid regardless of a particular DBMS data model and therefore not depend on it. ${ }^{2}$ Instead, the SDT definition should be based on an abstract interface to the DBMS data model which we call the object model interface.

The purpose of this paper (together with a companion paper) is to develop a formal definition of spatial data types fulfilling these criteria. The central idea is to introduce into the DBMS the concept of a realm. A realm is in general a finite, user defined structure that is used as a basis for one or more system data types. Realms are somewhat similar to enumeration types in programming languages. A

2 This also holds for the implementation level: A spatial type extension package (STEP) should be able to cooperate with any extensible DBMS offering a suitable interface regardless of its data model. 
realm used as a basis for spatial data types is essentially a finite set of points and non-intersecting line segments. Intuitively, it describes the complete underlying geometry of an application. All points, lines and regions associated with objects (from now on called spatial attribute values) can be defined in terms of points and line segments present in the realm. In fact, in a database spatial attribute values are then never created directly but only by selecting some realm objects. They are never updated directly. Instead, updates are performed on the realm and from there propagated to the dependent attribute values.

Hence, all attribute values occurring in a database are realm-based. Furthermore, the algebraic operations for the spatial data types are defined to construct only geometric objects that are realmbased as well. So the spatial algebra is closed with respect to a given realm. This means in particular that no two values of spatial data types occurring in geometric computation have "proper" intersections of line segments. Instead, two initially intersecting segments have already been split at the intersection point when they were entered into the realm. One could say that any two intersecting SDT values (say, lines or regions) "have become acquainted" already when they were entered into the realm. This is a crucial property for the correct and efficient implementation of geometric operations.

Realm objects - points and segments - are defined not in abstract Euclidean space but in terms of finite representations. All geometric primitives and realm operations (e.g. updates) are defined in error-free integer arithmetic. For mapping an application's set of intersecting line segments into a realm's set of non-intersecting segments the concept of redrawing and finite resolution geometry from [GrY86] is used. Although intersection points computed with finite resolution in general move away from their exact Euclidean position, this concept ensures that the unavoidable distortion of geometry (that is, the numerical error) remains bounded and very small and that essentially ${ }^{3}$ no topological errors occur. This means that a programmer has a precise specification that directly lends itself to a correct implementation. It also means that the spatial algebra obeys algebraic laws precisely in theory as well as in practice. Furthermore, it is rather obvious that realms also solve the geometric consistency problem.

Most closely related to this work are the formal definitions of spatial data types (or algebras) given by Güting [Gü88a, Gü88b], Scholl and Voisard [ScV89, Vo92], and Gargano et al. [GaNT91]. All of these proposals do not fulfill most of the criteria given above. In [Gü88a, Gï88b] data types for points, lines, and regions are available but too restricted, e.g. a region is a single simple polygon (without holes). In [ScV89] general regions are defined; in Voisard's thesis [Vo92] this has been extended to general types for points and lines. However, the definitions are unnecessarily complex. In [GaNT91] there is only a single type for all kinds of geometric objects; a value is essentially a set of sets of pixels. We feel this is not sufficient, since many interesting spatial operations cannot be expressed. As mentioned, all of these proposals give formal definitions. However, those of Güting and of Scholl and Voisard are not based on finite resolution; hence the numeric correctness problems are not addressed. Gargano et al. base their definitions in principle on a finite underlying set (of pixels). But this is not practical since these finite representations are far too large to be efficiently manageable. The geometric consistency problem is not solved in any of these proposals; there is some weak support in [Gü88a] through an AREA data type, but it is not sufficient. Finally, all three proposals have connected their spatial types to a fixed data model - Güting and Gargano et al. to the relational model

3 See the discussion in Sections 2 and 8. 
and Scholl and Voisard to a complex object algebra [AbB88]. Only Scholl and Voisard emphasize a clean interface between the spatial algebra and the general object model. We shall extend their work by offering an abstract interface not dependent on any particular data model.

The topological data model based on simplicial complexes suggested by Egenhofer et al. [EgFJ89] has a similar purpose as our concept of realms. Essentially they offer an irregular triangular network partition of the plane as a geometric domain over which spatial objects could be defined. However, the connections are missing to the underlying finite arithmetic as well as to spatial data types based on this model. Also, in our view a triangular partition contains too much information; it is sufficient to keep those points and segments in a geometric domain that are needed for spatial attribute values. Finally, their model is an abstract one whereas we show realms within a database context.

Our description and formal development of realm-based spatial data types is given in two papers. In this paper the lower layers, namely numerically robust geometric primitives, realms and their operations and the structure of values of the spatial data types are defined. In [GüS92] the object model interface and the spatial data types and operations, that is, the spatial algebra, are described. In the following section we first provide an informal overview of the complete concept.

\section{Overview: Realm-Based Spatial Data Types}

A realm is a set of points and non-intersecting line segments over a discrete domain, that is, a grid, as shown in Figure 1.

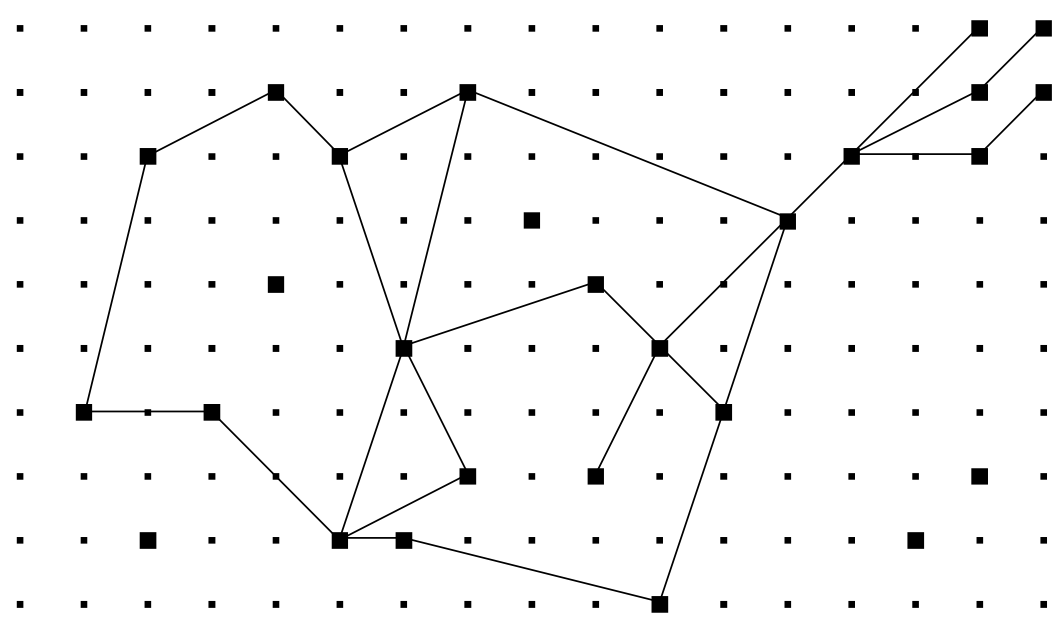

Figure 1

Values of spatial data types can be composed from the objects present in a realm. Figure 2 shows some values definable over the realm of Figure 1. Our realm-based spatial data types are called POINTS, LINES, and REGIONS, hence $\mathrm{A}$ and $\mathrm{B}$ represent REGIONS values, $\mathrm{C}$ is a LINES value, and $\mathrm{D}$ a POINTS value. The precise structure of these values is not yet relevant here. One can imagine A and $\mathrm{B}$ to belong to two adjacent countries, $\mathrm{C}$ to represent a river, and $\mathrm{D}$ a city.

The underlying grid of a realm arises simply from the fact that numbers have a finite representation in computer memory. In practice, these representations will be of fixed length and correspond to INTEGER or REAL data types available in programming languages. Of course, the resolution will be much finer than could be shown in Figure 1. 


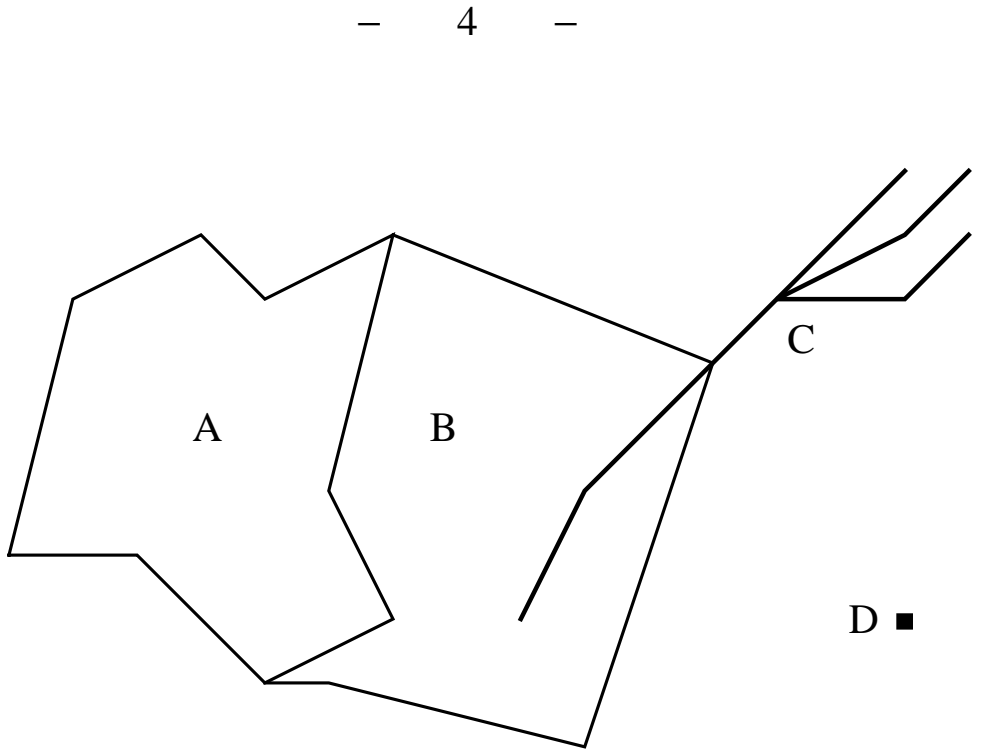

Figure 2

The concept of realm as a basis of spatial data types serves the following purposes:

- It enforces geometric consistency of related spatial objects. For example, the common part of the borders of countries A and B is exactly the same for both objects.

- It guarantees nice closure properties for the computation with spatial data types above the realm. For example, the intersection of region $\mathrm{B}$ with line $\mathrm{C}$ (the part of river $\mathrm{C}$ lying within country B) is also a realm-based LINES value.

- It shields geometric computation in query processing from numeric correctness and robustness problems. This is because such problems arise essentially from the computation of intersection points of line segments which normally do not lie on the grid. With realmbased SDTs, there are never any new intersection points computed in query processing. Instead, the numeric problems are treated below the realm level, namely, whenever updates are made to a realm.

- Additionally, a data structure representing a realm can be used as an index into the database. Our implementation concept assumes that each point and segment in a realm has an associated list of logical pointers to the spatial attribute values defined over it in the database.

Let us now focus on the treatment of numeric correctness problems below and within the realm level. This is necessary because geometric data coming from the application are not intersection-free, as required for a realm. Application data can at the lowest level of abstraction be viewed as a set of points and intersecting line segments. These need to be transformed into a realm. As mentioned before, the fundamental problem is that intersection points usually do not lie on the grid.

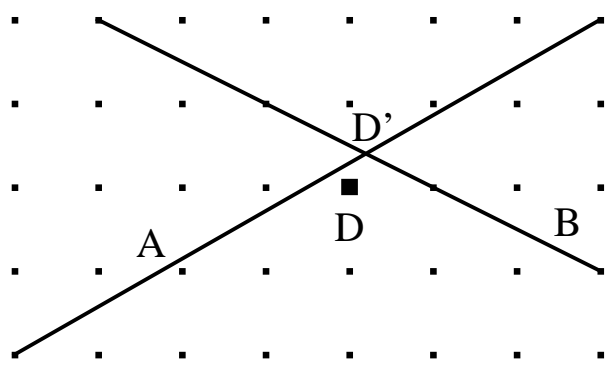

Figure 3

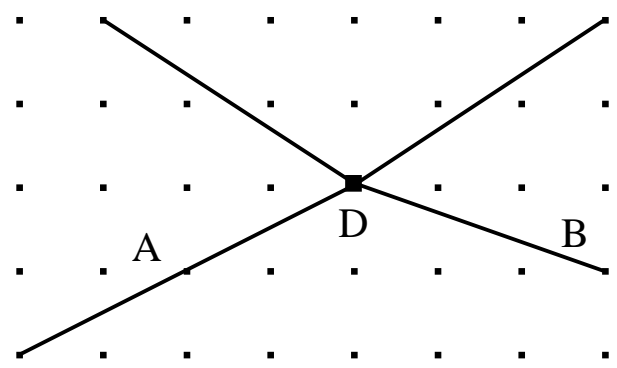

Figure 4

In Figure 3, the intersection point D' of line segments A and B will be moved to the closest grid point D. This leads, for example, to the following topological errors: (1) A test whether D lies on A or B 
fails. (2) A test whether D lies properly within some area defined below A and B will incorrectly yield true. (3) If there is another segment $\mathrm{C}$ between the true intersection point and $\mathrm{D}, \mathrm{D}$ will be reported to lie on the wrong side of $\mathrm{C}$. The basic idea to avoid these errors is to slightly change segments $\mathrm{A}$ and $B$ by transforming them into chains of segments going through $D$, as shown in Figure 4. However, this does not suffice, since it allows a segment to drift (through a series of intersections) by an arbitrary distance from its original position. For example, a further intersection of $\mathrm{A}$ with some segment $\mathrm{C}$ (Figure 5) is resolved as shown in Figure 6, where intersection point $\mathrm{E}$ has already a considerable distance from the true intersection point of $\mathrm{A}$ and $\mathrm{C}$. Note in particular, that segment $\mathrm{A}$ has in Figure 6 been moved to the other side of a grid point (indicated by the arrow) which may later be reported to lie on the wrong side of A.

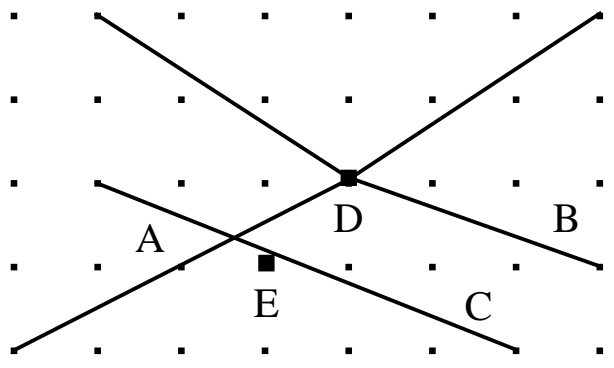

Figure 5

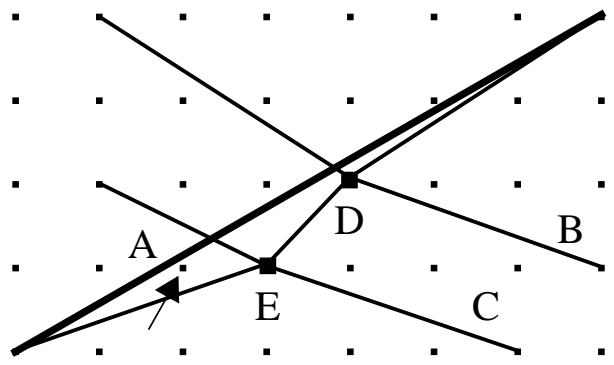

Figure 6

A refined solution was proposed by Greene and Yao [GrY86]. The idea is to define for a segment $s$ an envelope $E(s)$ roughly as the collection of grid points that are immediately above, below, or on $s$. An intersection of $s$ with some other segment may lead to a requirement that $s$ should pass through some point $P$ on its envelope (the grid point closest to the true intersection point). This requirement is then fulfilled by redrawing $s$ by some polygonal line within the envelope rather than by simply connecting $P$ with the start and end points of $s$. Figure 7 shows a segment $s$ (drawn fat) together with the grid points of its envelope. Slightly above $s$ a redrawing of $s$ through $P$ is shown.

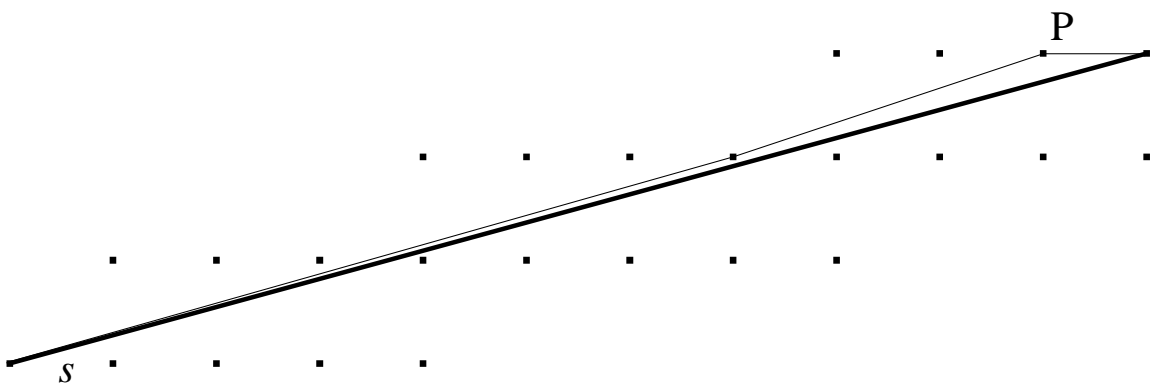

Figure 7

Intuitively, the process of redrawing can be understood as follows: Think of segment $s$ as a rubber band and the points of the envelope as nails on a board. Now grip $s$ at the true intersection point and pull it around $P$. The resulting polygonal path is the redrawing. The number of segments of this path is in the worst case logarithmic in the size of the grid, but it seems that in most cases only very few segments are created.

This approach guarantees that the polygonal line describing a segment always remains within the envelope of the original segment. We adopt the technique for realms. It then means that by redrawing a segment can never drift to the other side of a realm point. It might still happen, though, that after a redrawing a realm point is found to lie on a segment which it did not originally. 
The formal definition of realm-based SDTs is organized as a series of layers. Each layer defines its own structures and primitives, using the notions of the layers below. We describe these layers bottom-up in the rest of this paper and the companion paper [GüS92]. Let us briefly provide an overview of this development.

Robust geometric primitives are introduced in Section 3 (and an appendix). This lowest layer defines a discrete space $N \times N$ where $N=\{0, \ldots, n-1\}$ is a subset of the natural numbers. The objects in this space are points and line segments with coordinates in $N$, called $N$-points and $N$-segments. A number of operations (predicates) such as whether an $N$-point lies on an $N$-segment or whether two $N$-segments intersect, and which $N$-point is the result of intersecting two $N$-segments, are defined. The crucial point is that these definitions are given in terms of error-free integer arithmetic, hence they are directly implementable.

In Section 4 geometric realms are defined as described above; elements are called $R$-points and $R$-segments. Basic operations on realms (given in Section 5) are insertion and deletion of $N$-points and $\mathrm{N}$-segments. However, to cooperate with a database system, a realm - as an abstract data type - has a more general interface. For example, the operation of inserting an $N$-segment returns besides a modified realm a redrawing of the inserted segment and a set of redrawings of segments in the database that need to be modified together with logical pointers (SCIDs = spatial component identifiers) to database representations of these segments. The management of identifiers makes it necessary to offer operations that register spatial attribute values in the database with their underlying realm objects. A last group of operations allows to get a set of realm objects (within a rectangular window) and to identify objects when a close $N$-point is given. This makes it possible to display parts of a realm at a DBMS graphical user interface and to let a user build spatial attribute values by clicking at realm objects displayed.

The next layer (Section 6) defines certain structures present in a realm that serve as a basis for the definition of SDTs. A realm can be viewed as a planar graph; an $R$-cycle is a cycle of this graph. An $R$-face is an $R$-cycle possibly enclosing some other disjoint $R$-cycles corresponding to a region with holes. An $R$-unit is a minimal $R$-face. These three notions support the definition of a REGIONS data type. An $R$-block is a connected component of the realm graph; it supports the definition of a LINES data type. For all of these structures there are also predicates defined to describe their possible relationships.

After these preparations in the next layer (Section 7) the domains of spatial data types POINTS, LINES, and REGIONS are defined. A POINTS value is a set of $R$-points. There are two alternative views of LINES and REGIONS. The first is that a LINES value is a set of $R$-segments and a regions value a set of $R$-units. The other view is equivalent but "semantically richer": A lines value is a set of disjoint $R$-blocks and a regions value a set of (edge-) disjoint $R$-faces. This completes the scope of this paper.

In the companion paper [GüS92] first the notion of properties of collections of values is introduced. The purpose is to support notions such as a map which is essentially a set of objects whose REGIONS attribute values are disjoint. One would like to identify such constraints and to use them for the definition of algebra operations, e.g. map overlay. Next, the object model interface (OMI) is defined. We identify a number of concepts that need to be present in the DBMS data (or object) model to allow it to cooperate with our spatial algebra. For example, the OMI must offer a function that yields for a 
given object an associated SDT value, operations to form aggregates of objects or to group sets of objects by attribute values (which in connection with the spatial algebra allows to realize a "fusion" operation [ScV89, GaNT91]). The corresponding idea at the system level is that any extensible database system offering an OMI implementation can cooperate with a spatial type extension package $(S T E P)$ realizing the spatial algebra. Finally the spatial algebra itself is described; the semantics of all operations are formally defined. Due to the underlying realms, this algebra has simple and powerful operations, nice closure properties and observes a number of algebraic laws. It is straightforward to implement this algebra in such a way that the laws do also hold in practice.

\section{Robust Geometric Primitives}

The bottom layer of the formal development introduces a finite discrete space, points and line segments over this space, and some simple predicates and operations on them. Let $N=\{0, \ldots, n-1\}$ $\subseteq \mathbf{N}$. An $N$-point is a pair $(x, y) \in N \times N$. An $N$-segment is a pair of distinct $N$-points $(p, q)$. $P_{\mathrm{N}}$ denotes the set of all $N$-points and $S_{\mathrm{N}}$ the set of all $N$-segments. We introduce the primitives shown in Figure 8 on $N$-points and $N$-segments. In this graph representation, undirected edges denote predicates and directed edges other operations (here intersection takes two $N$-segments and returns an $N$-point).

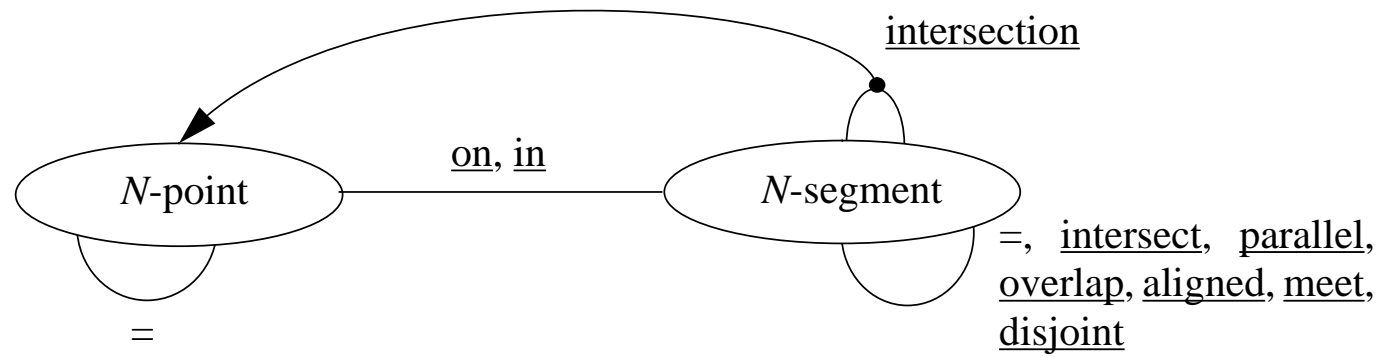

Figure 8

In the appendix these operations are defined in terms of integer arithmetic. Therefore they have a straightforward implementation free of numerical errors. We briefly explain them informally here: Two $N$-segments meet if they have exactly one end point in common. They overlap if they are collinear and share a (partial) $\mathrm{N}$-segment. If they are collinear and do not share a (partial) $\mathrm{N}$-segment, we call them aligned. If they have exactly one common point but do not meet, they intersect. If they have the same slope, they are parallel. They are disjoint if they are neither equal nor meet nor intersect. The on primitive tests if an $\mathrm{N}$-point lies on an $\mathrm{N}$-segment; the in primitive does nearly the same but the $N$-point must not coincide with one of the end points of the $N$-segment. The intersection primitive calculates the intersection point of two $N$-segments and rounds it to the nearest $N$-point.

\section{Realms}

Given $N$, a realm over $N$, or $N$-realm for short, is a set $R=P \cup S$ such that

(i) $P \subseteq P_{\mathrm{N}}, S \subseteq S_{\mathrm{N}}$

(ii) $\forall s \in S: s=(p, q) \Rightarrow p \in P \wedge q \in P$

(iii) $\forall p \in P \forall s \in S: \neg(p$ in $s)$

(iv) $\forall s, t \in S: \neg(s=t) \wedge \neg(s$ and $t$ intersect $) \wedge \neg(s$ and $t$ overlap) 
The elements of $P$ and $S$ are called $R$-points and $R$-segments. There is an obvious interpretation of a realm as a spatially embedded planar graph with set of nodes $P$ and set of edges $S$.

\section{Operations on Realms / The Realm Interface}

Obviously the fundamental operations on realms are the insertion of an $N$-point or $N$-segment and the deletion of an $R$-point or $R$-segment. However, the interface is a bit more complex since we study realms not just as abstract entities but in connection with spatial databases. That means that there are spatial attribute values in the database depending on realm objects. This dependency needs to be modeled and treated by the operations.

Our approach to implement the dependency is the following: We assume that (the geometry of) a spatial attribute value of some object is stored together with the object in the database and there is a logical pointer from each segment or point describing the spatial value to the underlying realm object. Furthermore, associated with each realm object (point or segment) is a set of logical pointers; one pointer to each corresponding component of a spatial value in the database. In other words, points and segments in the database are doubly linked with the corresponding points and segments in the realm. Pointers from the database into the realm are realized by realm object identifiers from a set ROID, pointers from the realm into the database by spatial component identifiers from a set SCID. It is assumed that each roid or scid uniquely identifies the corresponding entity and that the implementation guarantees fast access to these components. Additionally, a scid also identifies the spatial value as a whole.

This approach stores geometries redundantly with a database object and in the realm. One might save some space by representing attribute values just by structures composed of pointers to realm objects. However, we believe its crucial for efficient query processing and well worth the extra space to keep the geometries with the objects. In this way one can directly apply SDT operations to spatial attribute values whereas otherwise it would always be necessary to access (load pages of) the underlying realm. Note that the two-way linking is necessary in any case since changes in the realm (e.g. a new intersection point on a segment) need to be propagated to the dependent spatial values.

We model the management of pointers formally as follows: Let $R$ be a realm. A representation of $R$ is a set of triples $\{(r, \operatorname{roid}(r), \operatorname{scids}(r)) \mid r \in R\}$ where roid is a function giving for a realm object its unique identifier in ROID and scids is a function returning the set of SCIDs of dependent components of spatial attribute values. We also allow the notation roid(v) to assign a new ROID to a newly created realm object $v$.

The realm interface is described by the following signature. Slightly extending standard notations we allow operators to return tuples of values and sets of values - the type of a set of X values is denoted by $X^{*}$. The first group of operations are those mentioned above:

sorts Realm, Point, Segment, RealmObject, ROID, SCID, Rectangle, Bool, Integer

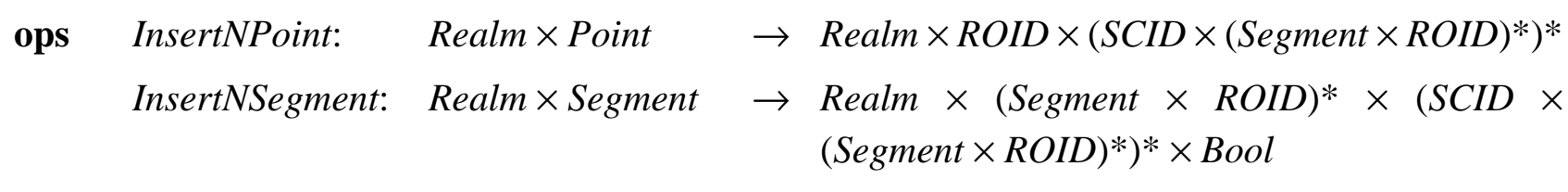

Delete: $\quad$ Realm $\times$ ROID $\rightarrow$ Realm $\times$ Bool 
The sort (type) Realm refers to a realm representation, Point to the set $P_{\mathrm{N}}$, Segment to the set $S_{\mathrm{N}}$. RealmObject is a union type of Point and Segment; we assume one can recognize whether a given instance is a point or a segment. ROID and SCID have been discussed above. Rectangle denotes the set of axis-parallel rectangles definable over space $N \times N$, that is, $N$-rectangles.

The update operations implement the approach of Greene and Yao [GrY86] described in Section 2 to preserve the topology for a set of intersecting line segments when they are represented over a finite grid. Greene and Yao do not deal with collections of points that are part of our realms. We extend their approach by an additional integrity rule for points and line segments that are very close to each other. In Section 2 the concept of an envelope was already introduced as a set of grid points "adjacent" to a segment (formally defined in [GrY86]). Let us call the "proper envelope" the subset of envelope points that are not end points of the segment (denoted $\bar{E}(s)$ for segment $s$ ). Then the rule is:

No R-point lies on the proper envelope of any $R$-segment.

The intuition behind this is that points that are so close are meant to lie on the segment. Update operations maintain this constraint by redrawing the segment whenever a point is discovered to lie on its proper envelope (which can happen on point insertion or on segment insertion).

The operation InsertNPoint takes a realm and an $N$-point. It returns (i) the modified realm, (ii) an identifier for the inserted point, which could be an old one if the point was in the realm already, and (iii) a set of segments in the database that need to be redrawn, which may be empty. A segment may need redrawing because the point lies on its proper envelope. For each such segment its "address" in the database (SCID) together with a list of pairs $(s, \operatorname{roid}(s))$ (where $s$ is a segment of the redrawing) is returned. It is then the task of the DBMS to replace segments by their redrawings.

The operation InsertNSegment takes a realm and an $\mathrm{N}$-segment. It returns (i) the modified realm, (ii) a list of segments with their roids which may contain either the original segment as the only element or a redrawing of this segment, (iii) a possibly empty set of segments that need to be redrawn (as for InsertNPoint). Here the inserted segment may need redrawing because it or its proper envelope touches $R$-points or because it intersects $R$-segments. The other segments need redrawing because they are intersected by this segment. The last parameter (iv) indicates whether insertion was performed; it was rejected, if not both end points of the segment were present in the realm.

The operation Delete takes a realm and the identifier of a realm object (point or segment) and removes the object from the realm if this doesn't violate certain integrity constraints. It returns (i) the modified realm and (ii) an indication whether the object was removed. The following conditions are checked: A point is only removed if there is no segment ending in the point. Any realm object is only removed if its set of scids (dependent objects) is empty.

The second group of operations supports the management of the two-way linking between realm objects and components of spatial values in the database:

$\begin{array}{lll}\text { Register: } & \text { Realm } \times \text { ROID } \times S C I D & \rightarrow \text { Realm } \\ \text { Unregister: } & \text { Realm } \times \text { ROID } \times S C I D & \rightarrow \text { Realm } \\ \text { GetSCIDs: } & \text { Realm } \times \text { ROID } & \rightarrow \text { SCID } \\ \text { GetRealmObject: } & \text { Realm } \times \text { ROID } & \rightarrow \text { RealmObject }\end{array}$


Here Register informs a realm object roid about a spatial component scid depending on it. Unregister removes such an information. GetSCIDs returns the scids of spatial components depending on the given roid, GetRealmObject returns the geometry. These operations are to be used, for example, as follows: A spatial attribute value is constructed by selecting a number of realm objects in a certain order (this is supported by the last group of operations, see below). After all components have been selected, the representation of this value is built and stored in the database. Then all components are registered with their underlying realm objects. When a spatial attribute value is deleted, the registration is removed for all objects. GetSCIDs and GetRealmObject are general purpose operations to support query processing.

The last group of operations supports the selection of realm objects for the construction of spatial values:

$$
\begin{aligned}
& \text { Window: } \quad \text { Realm } \times \text { Rectangle } \rightarrow(\text { RealmObject } \times \text { ROID })^{*} \\
& \text { Identify: } \quad \text { Realm } \times \text { Point } \times \text { Integer } \rightarrow \text { ROID } \times \text { Bool }
\end{aligned}
$$

Window returns all realm objects together with their roid that are inside or intersect a given rectangular window. Identify tries to identify a realm object close to the $N$-point given as a parameter. The number given as a third parameter controls the "pick distance". A roid (possibly undefined) is returned together with an indication whether identification was successful. These two operations can be used to retrieve a portion of a realm in order to define spatial attribute values over it. For example, this portion may be displayed at a graphical user interface. With a pointing device one can select $N$-points which through Identify determine realm objects from which the spatial values can be built.

We now define the semantics of the realm operations InsertNPoint and InsertNSegment. All the other operations are rather simple so that their meaning should be clear from the explanations above. InsertNPoint and InsertNSegment are described by giving algorithms for them. As mentioned before, the concepts of Greene and Yao [GrY86] are applied and extended, some of which need now to be explained in more detail. In [GrY86], for a set of line segments redrawings are computed in two phases. First, for each segment that needs to be redrawn, the set of points on its envelope, that need to be passed by the redrawing, is computed. These points are attached to the segment in the form of "hooks". A hook is a short directed line segment (an "arrow") from a point on the segment to the envelope point that needs to be passed.

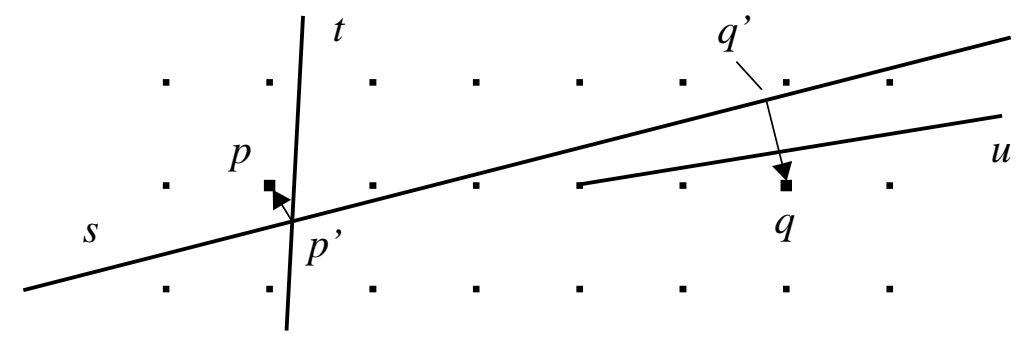

Figure 9

Figure 9 shows a segment $s$ with two hooks $\langle p$ ', $p\rangle$ and $\langle q$ ', $q\rangle$. Such a "hooked segment" is represented as a list $\left(s ;\left\langle p^{\prime}, p\right\rangle,\left\langle q^{\prime}, q\right\rangle\right)$. The first hook arises from the intersection of segments $s$ and $t$ in $p^{\prime}$; when this intersection is discovered, the hook $\left\langle p^{\prime}, p\right\rangle$ is added to both segments $s$ and $t$. Let us assume that the other hook in Figure 9 arises from the fact that point $q$ was inserted into the realm; 
since it lies on the envelope of $s, s$ should go through $q$. In such a case we take the point on the segment closest to the target point (in this case $q^{\prime}$ ) as the start point of the hook and denote it as base (<target>, <segment>), in this case $q^{\prime}=\operatorname{base}(q, s){ }^{4}$

Figure 9 also illustrates that for each hook created one generally needs to check whether any segments are intersected by it. In the example an intersection with segment $u$ would be discovered and a corresponding hook $\langle q ", q\rangle$ be added to segment $u$ (where $q$ " is the intersection point of the hook and $u$ ). Only after all hooks have been determined, redrawings (polygonal lines within the envelope) are computed. This can now be done for each hooked segment independently from all other segments. See [GrY86] for a description of how redrawings are computed.

The algorithm InsertNPoint has to treat the following cases: (i) the point is already present in the realm, (ii) the point is new and does not lie on any envelope, and (iii) the point falls on one or more proper envelopes. Only the last case is a bit more complex: All segments whose envelopes are touched get a hook. Later, all those segments are redrawn. The description uses predicates ExistsRPoint and ExistsRSegment with the obvious meaning to check whether a realm object to be created is present already.

algorithm InsertNPoint $\left(R, p, R^{\prime}, r, S P\right)$

\{Inputs are a realm $R=P \cup S$ and an $N$-point $p$. Outputs are the modified realm $R$, a realm object identifier $r$ for $p$, and a set $S P$ of spatial component identifiers and redrawings for the spatial objects which have to be updated.\}

Step 1

$S P:=\varnothing$

if $\exists q \in P: p=q$ (at most one such $R$-point can exist)

then $r:=\operatorname{roid}(q) ; R^{\prime}:=R$

else if $\forall s \in S: p \notin E(s)$

then $r:=\operatorname{roid}(p) ; R^{\prime}:=R \cup\{(p, r, \varnothing)\}$

else ( $R$-segments exist whose proper envelopes contain $p)$

$S_{\text {rd }}:=\varnothing$; (a set of $R$-segments which have to be redrawn)

$r:=\operatorname{roid}(p)$;

$S_{\text {env }}:=\{s \in S \mid p \in E(s)\}$

for each $s$ in $S_{\text {env }}$ do

Insert a hook $h=<$ base $(p, s), p>$ on $s ; S_{\text {rd }}:=S_{\text {rd }} \cup\{s\}$

(one does not need to check for segments in $S$ intersecting $h$ because they are already in $S_{\text {env }}$ )

Step 2 (redraw hooked lines)

Let $S_{\mathrm{rd}}:=\left\{t_{1}, \ldots, t_{\mathrm{n}}\right\}$. Let $\left\{t_{\mathrm{i}, 1}, \ldots, t_{\mathrm{i}, \mathrm{k}_{\mathrm{i}}}\right\}$ be the set of $k_{\mathrm{i}} R$-segments of the redrawing of $t_{\mathrm{i}}$ through $p$.

Let $t_{\mathrm{i}, \mathrm{j}}=\left(p_{\mathrm{i}, \mathrm{j}}, q_{\mathrm{i}, \mathrm{j}}\right), i \in\{1, \ldots, n\}, j \in\left\{1, \ldots, k_{\mathrm{i}}\right\}$

Step 3 (update realm)

$R^{\prime}:=R \backslash\left\{\left(t_{\mathrm{i}}, \operatorname{roid}\left(t_{\mathrm{i}}\right), \operatorname{scids}\left(t_{\mathrm{i}}\right)\right) \mid i \in\{1, \ldots, n\}\right\}$

(Insert the end points of the $R$-segments of the redrawings and the $R$-segments themselves if they do not already exist in the realm)

4 In [GrY86] all hooks arise from segment intersections. 


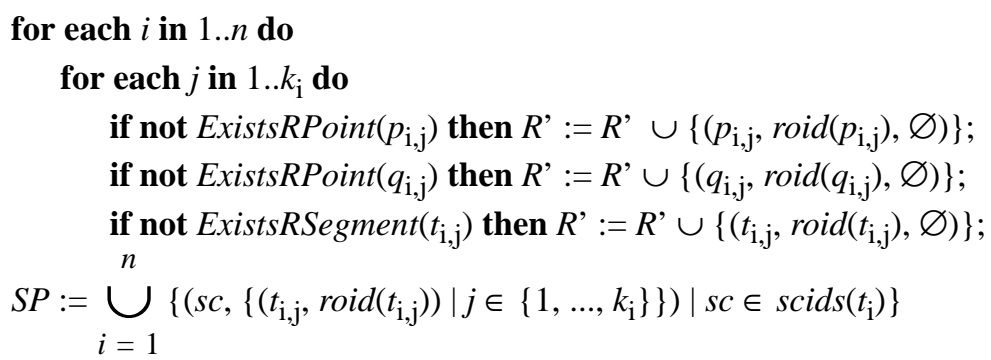

end InsertNPoint.

The algorithm InsertNSegment first checks whether the end points of the segment are present in the realm; otherwise it rejects insertion. This agrees with the graph-theoretic view of a realm: An edge can only exist if its nodes are there. It implies that the user of the realm layer has to make sure that the points are present (in case of doubt just insert them first; this doesn't hurt). Hence, when a segment is inserted, it is known that the end points have already interacted properly with envelopes of other segments. The following cases are now distinguished: (i) the segment is in the realm already, (ii) the segment is new and doesn't touch anything, and (iii) the segment may intersect some other segments and / or its envelope touches some realm points.

algorithm InsertNSegment $\left(R, s, R^{\prime}, R D, S P, o k\right)$

\{Inputs are a realm $R=P \cup S$ and an $N$-segment $s$. Outputs are the modified realm $R$, a set $R D$ of pairs of $R$-segments and realm object identifiers either for $s$ or a redrawing of $s$, a set $S P$ of spatial component identifiers and redrawings for the spatial objects which have to be updated, and a parameter $o k$ which indicates whether the insertion was performed. Insertion was rejected if the end points of $s$ were not present in the realm.

Step 1 (initializations)

$$
S P:=\varnothing ;
$$

Step 2 (check, whether end points of $s$ have already been inserted)

Let $s=\left(q_{1}, q_{2}\right)$. ok $:=$ ExistsRPoint $\left(q_{1}\right)$ and ExistsRPoint $\left(q_{2}\right)$;

if $o k$ then (execute steps 3-5)

Step 3 (insert hooks)

if $\exists t \in S: s=t$ (at most one such $R$-segment can exist)

then $R^{\prime}:=R ; R D:=\{(s, \operatorname{roid}(t))\}$

else if $\forall t \in S: s$ and $t$ are disjoint $\wedge \forall p \in P: p \notin E(s)$

then $R^{\prime}:=R \cup\{(s, \operatorname{roid}(s), \varnothing)\} ; R D:=\{(s, \operatorname{roid}(s))\}$

else ( $s$ intersects $R$-segments and/or there are $R$-points in the proper envelope of $s$ )

$S_{\text {rd }}:=\varnothing$ (the set of $R$-segments which have to be redrawn)

(Get all $R$-points lying in the proper envelope of $s$ except for the end points. Get all $R$-segments intersecting $S$.)

$P_{\text {env }}(s):=\{p \in P \mid p \in E(s)\}$

$S_{\text {intersect }}(s):=\{t \in S \mid s$ and $t \underline{\text { intersect }}\}$

for each $p$ in $P_{\text {env }}(s)$ do

Insert a hook $h=\langle\operatorname{base}(p, s), p\rangle$ on $s$

for each $t$ in $S$ do

if $h$ and $t$ intersect at $p$,

then

Insert a hook $h=\left\langle p^{\prime}, p>\right.$ on $t ; S_{\text {rd }}:=S_{\text {rd }} \cup\{t\}$ 


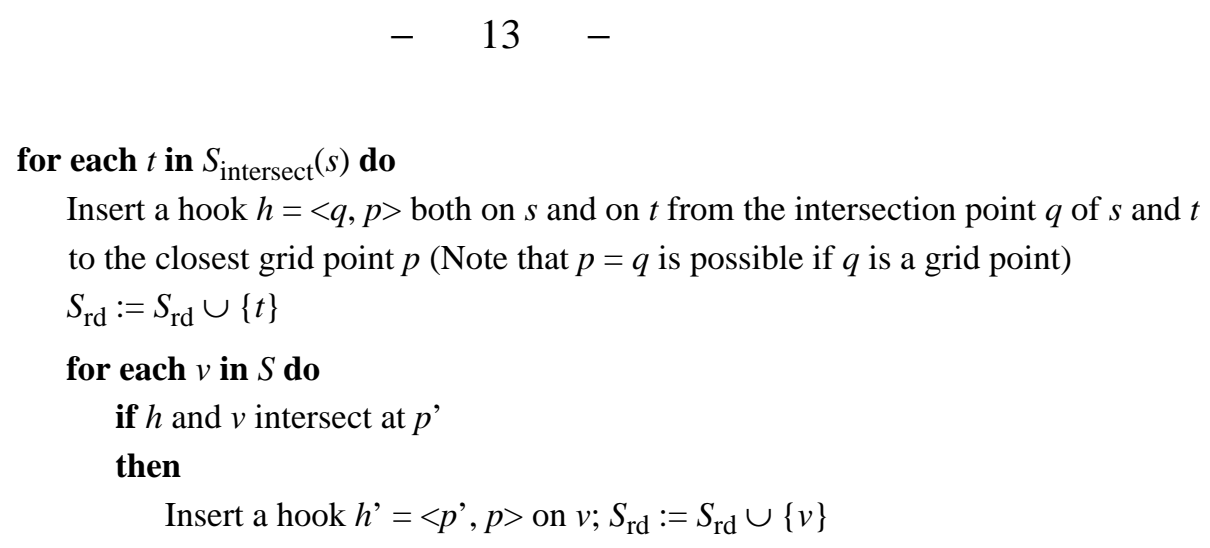

Insert a hook $h=\langle q, p\rangle$ both on $s$ and on $t$ from the intersection point $q$ of $s$ and $t$ to the closest grid point $p$ (Note that $p=q$ is possible if $q$ is a grid point)

$S_{\text {rd }}:=S_{\text {rd }} \cup\{t\}$

for each $v$ in $S$ do

if $h$ and $v$ intersect at $p$,

then

Insert a hook $h^{\prime}=\left\langle p^{\prime}, p>\right.$ on $v ; S_{\text {rd }}:=S_{\text {rd }} \cup\{v\}$

Step 4 (redraw hooked lines)

Redraw $s$. Let $\left\{s_{1}, \ldots, s_{\mathrm{m}}\right\}$ be the $R$-segments of the redrawing of $s$. Let $s_{\mathrm{i}}=\left(p_{\mathrm{i}}, q_{\mathrm{i}}\right), i \in\{1, \ldots, m\}$. Redraw all $R$-segments of $S_{\text {rd }}:=\left\{t_{1}, \ldots, t_{\mathrm{n}}\right\}$. Let $\left\{t_{\mathrm{i}, 1}, \ldots, t_{\mathrm{i}, \mathrm{k}}\right\}$ be the set of $k_{\mathrm{i}} R$-segments of the redrawing of $t_{\mathrm{i}}$. Let $t_{\mathrm{i}, \mathrm{j}}=\left(p_{\mathrm{i}, \mathrm{j}}, q_{\mathrm{i}, \mathrm{j}}\right), i \in\{1, \ldots, n\}, j \in\left\{1, \ldots, k_{\mathrm{i}}\right\}$

Step 5 (update realm)

$R^{\prime}:=R \backslash\left\{\left(t_{\mathrm{i}}, \operatorname{roid}\left(t_{\mathrm{i}}\right), \operatorname{scids}\left(t_{\mathrm{i}}\right)\right) \mid i \in\{1, \ldots, n\}\right\}$

(Insert the end points of the R-segments of the redrawings and the R-segments themselves if they do not already exist in the realm.)

for each $i$ in $1 . . m$ do

if not $\operatorname{ExistsRPoint}\left(p_{\mathrm{i}}\right)$ then $R^{\prime}:=R^{\prime} \cup\left\{\left(p_{\mathrm{i}}, \operatorname{roid}\left(p_{\mathrm{i}}\right), \varnothing\right)\right\}$

if not ExistsRPoint $\left(q_{\mathrm{i}}\right)$ then $R^{\prime}:=R^{\prime} \cup\left\{\left(q_{\mathrm{i}}, \operatorname{roid}\left(q_{\mathrm{i}}\right), \varnothing\right)\right\}$

if not ExistsRSegment $\left(s_{\mathrm{i}}\right)$ then $R^{\prime}:=R^{\prime} \cup\left\{\left(s_{\mathrm{i}}, \operatorname{roid}\left(s_{\mathrm{i}}\right), \varnothing\right)\right\}$

for each $i$ in $1 . . n$ do

for each $j$ in $1 . . k_{\mathrm{i}}$ do

if not ExistsRPoint $\left(p_{\mathrm{i}, \mathrm{j}}\right)$ then $R^{\prime}:=R^{\prime} \cup\left\{\left(p_{\mathrm{i}, \mathrm{j}}, \operatorname{roid}\left(p_{\mathrm{i}, \mathrm{j}}\right), \varnothing\right)\right\}$

if not ExistsRPoint $\left(q_{\mathrm{i}, \mathrm{j}}\right)$ then $R^{\prime}:=R^{\prime} \cup\left\{\left(q_{\mathrm{i}, \mathrm{j}}, \operatorname{roid}\left(q_{\mathrm{i}, \mathrm{j}}\right), \varnothing\right)\right\}$

if not ExistsRSegment $\left(t_{\mathrm{i}, \mathrm{j}}\right)$ then $R^{\prime}:=R^{\prime} \cup\left\{\left(t_{\mathrm{i}, \mathrm{j}}, \operatorname{roid}\left(t_{\mathrm{i}, \mathrm{j}}\right), \varnothing\right)\right\}$

$R D:=\left\{\left(s_{\mathrm{i}}, \operatorname{roid}\left(s_{\mathrm{i}}\right)\right) \mid i \in\{1, \ldots, m\}\right\}$

$S P:=\bigcup_{i=1}^{n}\left\{\left(s c,\left\{\left(t_{\mathrm{i}, \mathrm{j}}, \operatorname{roid}\left(t_{\mathrm{i}, \mathrm{j}}\right)\right) \mid j \in\left\{1, \ldots, k_{\mathrm{i}}\right\}\right\}\right) \mid \operatorname{sc} \in \operatorname{scids}\left(t_{\mathrm{i}}\right)\right\}$

end InsertNSegment.

\section{Realm-Based Structures and Primitives}

We can now assume that the problems of numerical robustness and topological correctness are solved by the lower layers. Given is a realm which can be viewed as a planar graph over the grid $N \times N$. Within a realm one can discover certain structures and relationships between these structures useful for the definition of spatial data types. These structures are called $R$-cycle, $R$-face, $R$-unit, and $R$-block. For the relationships we will define a number of predicates (primitives).

An $R$-cycle $c$ is just a cycle in the graph interpretation of a realm, defined by a set of $R$-segments $S(c)=\left\{s_{1}, \ldots, s_{\mathrm{m}}\right\}$, such that

(i) $\quad \forall i \in\{1, \ldots, m\}: s_{\mathrm{i}}$ meets $s_{(\mathrm{i}+1) \bmod \mathrm{m}}$

(ii) No other pairs of segments in $S(c)$ meet.

Obviously the following relationships may exist between an $N$-point $p$ and an $R$-cycle $c$ : 
(i)

$p \underline{\text { on }} c \quad: \Leftrightarrow \quad \exists s \in S(c): p$ on $s$

For $p=(x, y)$ let $s_{\mathrm{p}}=((x, y),(x, n-1))$ (that is, a vertical segment extending from $p$ upwards to the edge of the grid). Let $S_{\mathrm{r}}(c)$ be the segments in $S(c)$ whose right end point, but not the left one, is on $s_{\mathrm{p}}$. Let $S_{\mathrm{i}}(c)$ be the segments in $S(c)$ that intersect $s_{\mathrm{p}}$. Then

(ii) $\quad p$ inc $\quad: \Leftrightarrow \neg p$ on $c \wedge\left|S_{\mathrm{r}}(c)\right|+\left|S_{\mathrm{i}}(c)\right|$ is odd $^{5}$

(iii) $p \underline{\text { out }} c: \Leftrightarrow \neg(p \underline{\text { on }} c \vee p \underline{\text { in }} c)$

Hence $c$ partitions the set $P_{\mathrm{N}}$ into three subsets $P_{\text {in }}(c), P_{\text {on }}(c)$, and $P_{\text {out }}(c)$. Let $P(c):=P_{\text {on }}(c) \cup P_{\text {in }}(c)$.

Cycles are interesting because they are the basic entities for the definition of regions over realms. The relationships shown in Figure 10 may be distinguished between two $R$-cycles $c_{1}$ and $c_{2}$ :
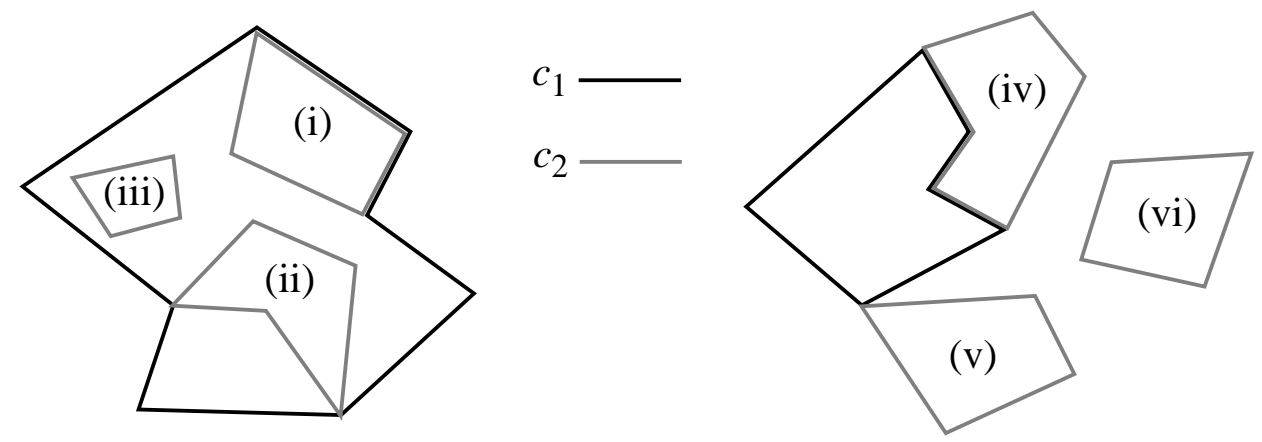

Figure 10

We introduce the following terminology for these configurations:

$c_{2}$ is

- (area-)inside (i, ii, iii)

- edge-inside (ii, iii)

- vertex-inside (iii) $c_{1}$ and $c_{2}$ are

- area-disjoint (iv, v, vi)

- edge-disjoint (v, vi)

- (vertex-)disjoint (vi)

$c_{1}$

The meaning is that (i) $c_{2}$ is (w.r.t area) inside $c_{1}$, (ii) additionally has no common edges with $c_{1}$, (iii) has not even common vertices with $c_{1}$. Similarly (iv) $c_{2}$ is disjoint w.r.t. area with $c_{1}$, (v) additionally has no common edges with $c_{1}$, (vi) additionally has not even common vertices with $c_{1}$. area-inside is the standard interpretation of the term inside, vertex-disjoint the standard interpretation of the term disjoint.

Furthermore there are two positive notions: $c_{1}$ and $c_{2}$ are adjacent if they are area-disjoint and have common edges, they meet if they are area-disjoint and have common vertices. These predicates are formally defined as follows:

$$
\begin{array}{ll}
c_{1} \text { ( } \underline{\text { area- }) \text { inside }} c_{2} & : \Leftrightarrow P\left(c_{1}\right) \subseteq P\left(c_{2}\right) \\
c_{1} \text { edge-inside } c_{2} & : \Leftrightarrow c_{1} \text { area-inside } c_{2} \wedge S\left(c_{1}\right) \cap S\left(c_{2}\right)=\varnothing \\
c_{1} \text { vertex-inside } c_{2} & : \Leftrightarrow c_{1} \underline{\text { edge-inside }} c_{2} \wedge P_{\text {on }}\left(c_{1}\right) \cap P_{\text {on }}\left(c_{2}\right)=\varnothing \\
c_{1} \text { and } c_{2} \text { are } \underline{\text { area-disjoint }} & : \Leftrightarrow P_{\text {in }}\left(c_{1}\right) \cap P\left(c_{2}\right)=\varnothing \wedge P_{\text {in }}\left(c_{2}\right) \cap P\left(c_{1}\right)=\varnothing
\end{array}
$$

5 This is a precise grid-based formulation of the well-known "plumbline" algorithm. 


$$
\begin{array}{lll}
c_{1} \text { and } c_{2} \text { are } \underline{\text { edge-disjoint }} & : \Leftrightarrow c_{1} \text { and } c_{2} \text { are } \underline{\text { area-disjoint }} \wedge S\left(c_{1}\right) \cap S\left(c_{2}\right)=\varnothing \\
c_{1} \text { and } c_{2} \text { are }(\underline{\text { vertex- }) \underline{\text { disjoint }}} & : \Leftrightarrow c_{1} \text { and } c_{2} \text { are } \underline{\text { edge-disjoint }} \wedge P_{\text {on }}\left(c_{1}\right) \cap P_{\text {on }}\left(c_{2}\right)=\varnothing \\
& \text { (which is equivalent to saying that } \left.P\left(c_{1}\right) \cap P\left(c_{2}\right)=\varnothing\right) \\
c_{1} \text { and } c_{2} \text { are } \underline{\text { adjacent }} & : \Leftrightarrow c_{1} \text { and } c_{2} \text { are } \underline{\text { area-disjoint }} \wedge S\left(c_{1}\right) \cap S\left(c_{2}\right) \neq \varnothing \\
c_{1} \text { and } c_{2} \underline{\text { meet }} & : \Leftrightarrow c_{1} \text { and } c_{2} \text { are } \underline{\text { edge-disjoint }} \wedge P_{\text {on }}\left(c_{1}\right) \cap P_{\text {on }}\left(c_{2}\right) \neq \varnothing
\end{array}
$$

One can observe similar ways how an $R$-segment $s$ can lie within an $R$-cycle $c$ :

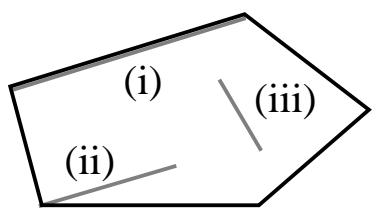

- $\quad s(\underline{\text { area }}$-) $\underline{\text { inside }} c(\mathrm{i}, \mathrm{ii}, \mathrm{iii})$

- $s \underline{\text { edge-inside }} c$ (ii, iii)

- $\quad s \underline{\text { vertex-inside }} c$ (iii)

Figure 11

For an $R$-point $p$ and an $R$-cycle $c$ we have two possibilities:

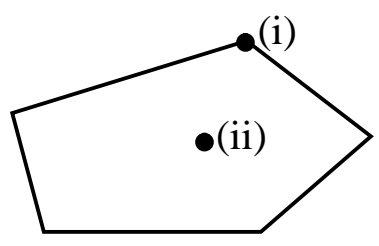

- $p(\underline{\text { area- }}) \underline{\text { inside }} c$ (i, ii)

- $\quad p$ vertex-inside $c$ (ii)

Figure 12

Formal definitions are left to the reader.

Based on the concept of $R$-cycles, for the definition of a spatial data type for regions the notions $R$-face and $R$-unit are introduced which describe regions from two different perspectives and which are used equivalently. Both of them essentially define polygonal regions with holes. An $R$-unit is a "minimal" $R$-face in the sense that any $R$-face within the $R$-unit is equal to the $R$-unit. Hence $R$-units are the smallest region entities that exist over a realm. We will see that any two $R$-units are area-disjoint and that any $R$-face can be described as a set of $R$-units. In the next section a region (data type) will be defined that can either be viewed as a set of $R$-faces or, equivalently, as a set of $R$-units. The first view emphasizes a minimal representation of the boundary of a region whereas the latter view supports the definition of set operations for regions. We will define operations to convert between the two (formal) representations.

Let $C(R)$ denote the set of all $R$-cycles. An $R$-face $f$ is a pair $(c, H)$ where $c$ is an $R$-cycle and $H=$ $\left\{h_{1}, \ldots, h_{\mathrm{m}}\right\}$ is a (possibly empty) set of $R$-cycles such that the following conditions hold (let $S(f)$ denote the set of all segments of all cycles of $f$ ):

(i) $\forall i \in\{1, \ldots, m\}: h_{\mathrm{i}} \underline{\text { edge-inside }} c$

(ii) $\forall i, j \in\{1, \ldots, m\}, i \neq j: h_{\mathrm{i}}$ and $h_{\mathrm{j}}$ are $\underline{\text { edge-disjoint }}$

(iii) Each cycle in $S(f)$ is either equal to $c$ or to one of the cycles in $H$ (no other cycle can be formed from the segments of $f$ )

The last condition ensures uniqueness of representation, that is, there are no two different 
interpretations of a set of segments as sets of faces. For example, it guarantees that the configuration shown in Figure 13 must be interpreted as two faces, and not as a single face with 5 holes (since under the latter interpretation the cycle drawn fat would violate condition (iii)).

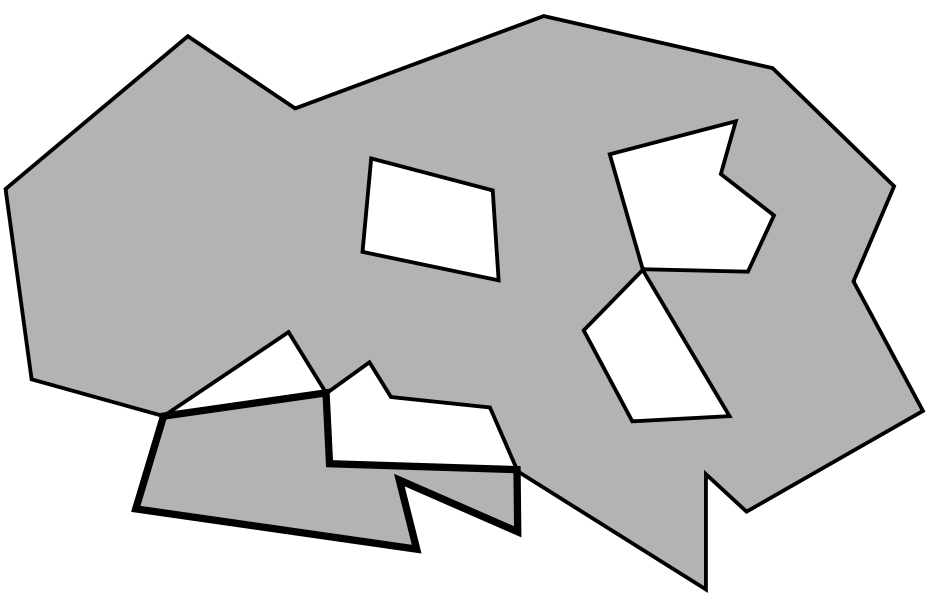

Figure 13

With terms defined below condition (iii) can be rephrased as "an $R$-face cannot be decomposed into two or more edge-disjoint $R$-faces".

The grid points belonging to an $R$-face $f$ are defined as:

$P(f):=P(c) \backslash \bigcup_{i=1}^{m} P_{\text {in }}\left(h_{\mathrm{i}}\right)$

Let $S(F)$ denote the set of all $R$-segments of a set of $R$-faces $F$.

The possible relationships between an $R$-point $p$ or an $R$-segment $s$ and an $R$-face $f=(c, H)$ are:

(i) $\quad p$ (area-) $\underline{\text { inside } f}: \Leftrightarrow p$ area-inside $c \wedge \forall h \in H: \neg p$ vertex-inside $h$

(ii) $\quad s$ (area-)inside $f: \Leftrightarrow s$ area-inside $c \wedge \forall h \in H: \neg s$ edge-inside $h$

The various notions of inside and $\underline{\text { disjoint }}$ can be extended for the comparison of two $R$-faces $f=\left(f_{0}, \bar{F}\right)$ and $g=\left(g_{0}, \bar{G}\right)$, for example:

$f$ (area-) $\underline{\text { inside }} g \quad: \Leftrightarrow f_{0}$ area-inside $g_{0} \wedge \forall \bar{g} \in \bar{G}: \bar{g}$ area-disjoint $f_{0} \vee \exists \bar{f} \in \bar{F}: \bar{g}$ area-inside $f$

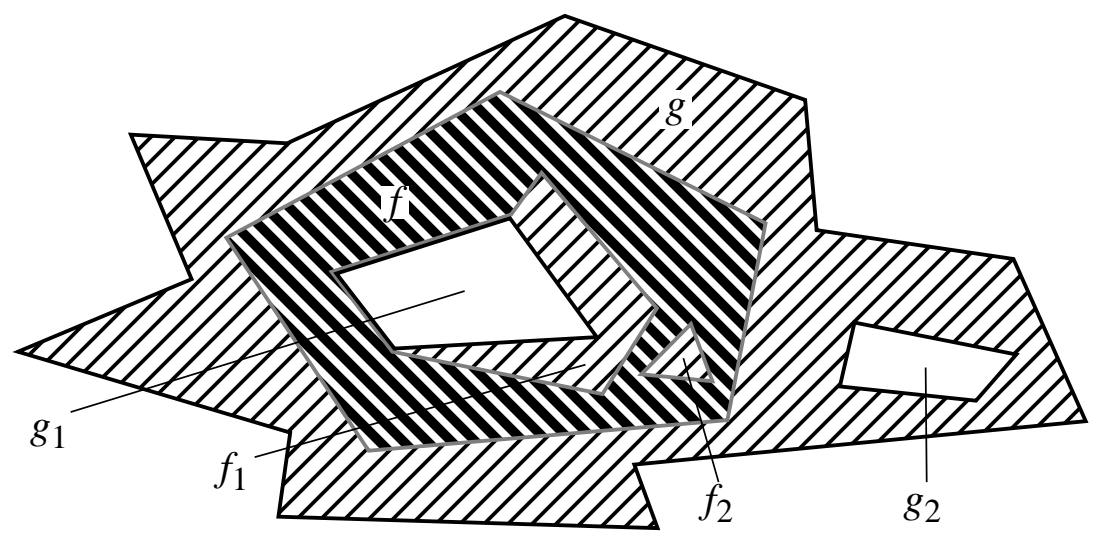

Figure 14

This definition is illustrated in Figure 14. 
f area-disjoint $g \quad: \Leftrightarrow f_{0}$ area-disjoint $g_{0} \vee \exists \bar{g} \in \bar{G}: f_{0}$ area-inside $\bar{g} \vee \exists \bar{f} \in \bar{F}: g_{0}$ area-inside $\bar{f}$ f $\underline{\text { edge-disjoint } g}: \Leftrightarrow f_{0}$ edge-disjoint $g_{0} \vee \exists \bar{g} \in \bar{G}: f_{0}$ edge-inside $\bar{g} \vee \exists \bar{f} \in \bar{F}: g_{0}$ edge-inside $\bar{f}$

The meaning of the remaining predicates edge-inside, vertex-inside, vertex-disjoint, adjacent, meet should be clear; definitions are omitted for brevity.

An $R$-unit as a minimal $R$-face is defined as follows. Let $F(R)$ denote the set of all possible $R$-faces. Let $f$ be an $R$-face.

$f$ is an $R$-unit $\quad: \Leftrightarrow \forall g \in F(R): g$ area-inside $f \Rightarrow g=f$

We also denote by $U(R)$ the set of all $R$-units.

Our goal is now to establish an equivalence between two representations of a region over a realm, namely, as a set of (pairwise) edge-disjoint $R$-faces, and as a set of $R$-units. First we consider the conversion of a set of faces into a set of units. We need two lemmas, whose proofs are technical and are only sketched:

Lemma 6-1 Let $f$ be an $R$-face and $u$ an $R$-unit. Then either $u$ area-inside $f$ or $u$ area-disjoint $f$.

The idea of the proof is that if this is not the case, then one of the cycles of $f$, say $f^{\prime}$, must properly intersect one of the cycles of $u$, say $u$ '.

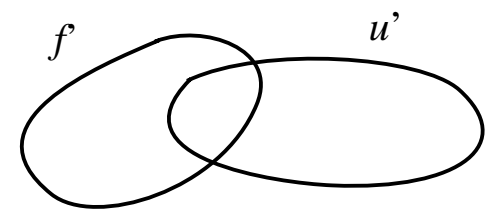

Figure 15

But then a part of $f^{\prime}$ lies within $u$ and forms a cycle there with a part of $u$ '. Hence there would be a face contained in $u$ different from $u$ which contradicts the definition of an $R$-unit.

Lemma 6-2 Let $f$ be an $R$-face and $u$ an $R$-unit such that $u$ area-inside $f$. Then "subtracting" $u$ from $f$ results in a set of $R$-faces.

The idea of the proof is the following: If $u$ is even edge-inside $f$ then removing the area of $u$ from $f$ just adds another hole to $f$. If $u$ 's outer cycle $u_{0}$ has some adjacent parts with $f$ 's outer cycle $f_{0}$, then a

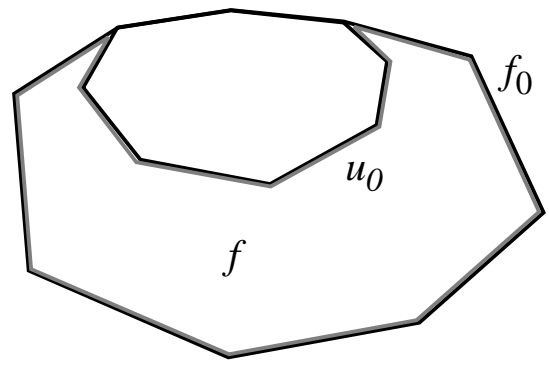

Figure 16

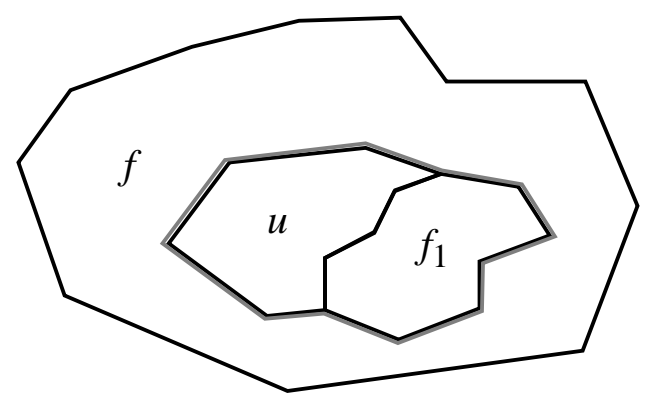

Figure 17

"bay" is formed in $f_{0}$ (Figure 16). If it is adjacent with a hole $f_{1}$ in $f$, then $f_{1}$ will grow (Figure 17). If several adjacencies are present, then $f$ may be decomposed into several faces. 
The second lemma implies that the units inside a face $f$ cover the area of $f$ completely. For, if some area were left, it would form its own face which could again be decomposed into units.

Therefore the following definition correctly decomposes faces into units. Let $F$ be a set of edgedisjoint $R$-faces.

$$
\operatorname{units}(F):=\{u \in U(R) \mid \exists f \in F: u \text { area-inside } f\}
$$

We now consider the conversion of a set of units into a set of faces. Given a set of $R$-segments $S$, we say, $S$ describes a set of pairwise edge-disjoint $R$-faces $: \Leftrightarrow$ there exists a set of edge-disjoint $R$-faces $F$ such that $S=S(F)$. Furthermore, let $\Delta$ denote the operator for symmetrical set difference, that is, $V \Delta W=(V \backslash W) \cup(W \backslash V) . \Delta$ forms the union of two sets removing their intersection. The operator is associative and commutative. The basis for the conversion is the following lemma:

Lemma 6-3 Let $f$ and $g$ be two area-disjoint $R$-faces. Then $S(f) \Delta S(g)$ describes a set of edge-disjoint $R$-faces.

The basic idea is that the $\Delta$ operator just removes segments that are common to both faces. The areadisjointness condition makes sure that only boundaries between adjacent areas are removed (and not boundaries between a covered region in one face and a hole in the other face).

The lemma can be extended to two sets of faces: Let $F, G$ be two sets of edge-disjoint $R$-faces such that the faces in $F \cup G$ are pairwise area-disjoint. Then $S(F) \Delta \mathrm{S}(G)$ describes a set of edge-disjoint $R$-faces. Let the resulting set of $R$-faces be denoted as $F+G$. Now the conversion from units to faces can be defined as follows. Let $U$ be a set of $R$-units.

$$
\operatorname{faces}(U)=\sum_{u \in U}\{u\}
$$

We summarise the equivalence in

Theorem 6-4 $\forall F \subseteq F(R)$ : faces $($ units $(F))=F$

For the definition of a spatial data type for lines the notion of an $R$-block is introduced. A set $S$ of $R$-segments is called connected $: \Leftrightarrow \forall r, t \in S \exists s_{1}, \ldots, s_{\mathrm{m}}, s_{\mathrm{i}} \in S: r=s_{1}, t=s_{\mathrm{m}}$, and $\forall i \in\{1, \ldots, m-$ 1\} : $s_{\mathrm{i}}$ and $s_{\mathrm{i}+1}$ meet. An $R$-block is a connected set of $R$-segments. Two $R$-blocks $b_{1}$ and $b_{2}$ are disjoint $: \Leftrightarrow \forall s_{1} \in S\left(b_{1}\right) \forall s_{2} \in S\left(b_{2}\right): s_{1}$ and $s_{2}$ are disjoint. For an $R$-point $p$ we consider the angularly sorted cyclic list $L_{\mathrm{p}}$ of $R$-segments $s \in S\left(b_{1}\right) \cup S\left(b_{2}\right)$ that meet in $p$. $p$ is called a meeting point if $L_{\mathrm{p}}$ can be subdivided into two sublists $L_{\mathrm{p}, 1}$ and $L_{\mathrm{p}, 2}$ (whose concatenation leads to $L_{\mathrm{p}}$ ) so that all $R$-segments of $L_{\mathrm{p}, 1}$ are elements of $S\left(b_{1}\right)$ and all $R$-segments of $L_{\mathrm{p}, 2}$ are elements of $S\left(b_{2}\right)$, or vice versa (see Figure 18).
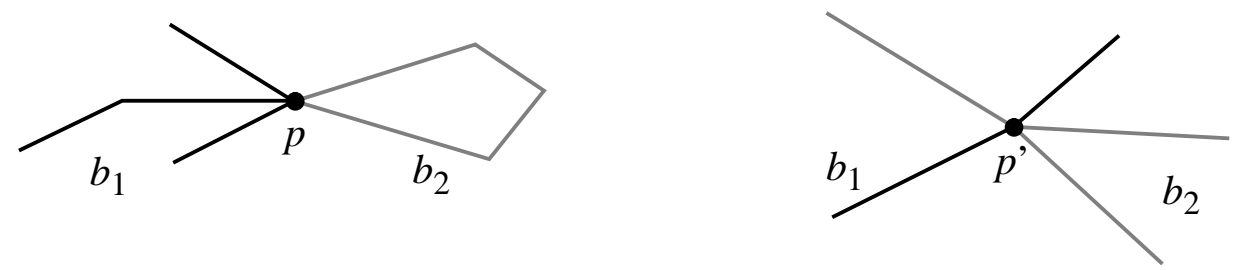

Figure 18: $p$ is a meeting point, $p$ ' is not a meeting point. 
Two $R$-blocks $b_{1}$ and $b_{2}$ meet $: \Leftrightarrow$

$$
\forall s \in S\left(b_{1}\right) \forall t \in S\left(b_{2}\right): s \neq t
$$

(ii) $\forall s \in S\left(b_{1}\right) \forall t \in S\left(b_{2}\right): s$ and $t \underline{\text { meet }} \Rightarrow s$ and $t$ meet in a meeting point.

Again, we have two equivalent representations of a lines value, namely, as a set of segments, or as a set of disjoint $R$-blocks. For a set of segments $S^{\prime} \subseteq S$ let blocks $\left(S^{\prime}\right)$ denote its partition into maximal connected components. Then $S\left(\operatorname{blocks}\left(S^{\prime}\right)\right)=S^{\prime}$.

\section{Realm-Based Spatial Data Types}

A formal definition of realm-based spatial data types (including operations) is given in the companion paper [GüS92]. However, to conclude this paper we show the connection between the realm-based structures of the previous section and the domains of the spatial data types. The basic types introduced are called POINTS, LINES, and REGIONS. There is a "flat" and a "structured" view of values of these types. The "flat" view is the following:

For a given realm $R$, a value of type POINTS is a set of R-points, a value of type LINES is a set of $R$-segments, and a value of type REGIONS is a set of $R$-units.

The structured view, that we shall assume as the formal definition, is as follows:

For a given realm $R$, a value of type POINTS is a set of R-points, a value of type LINES is a set of pairwise disjoint $R$-blocks, and a REGIONS value is a set of pairwise edge-disjoint $R$-faces.

We have shown in the previous section that the two views are equivalent. The first view is conceptually very simple and supports a direct understanding of set operations. The second view shows LINES and REGIONS values as consisting of a number of components (blocks or faces), allows one to express relationships between these components and also emphasizes the representation of the boundary in case of regions. Figure 19 illustrates the data types.

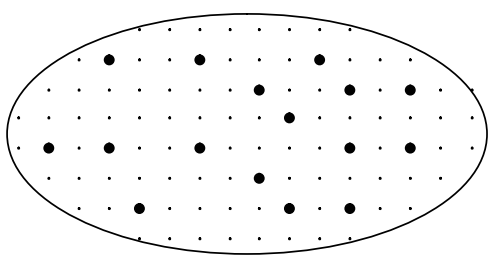

a POINTS value

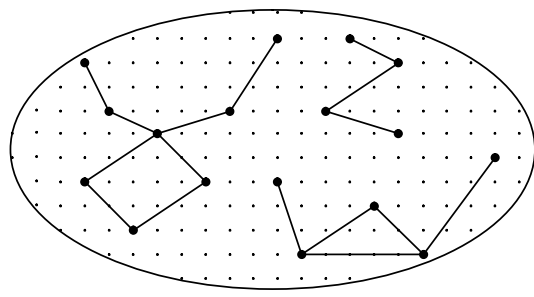

a LINES value

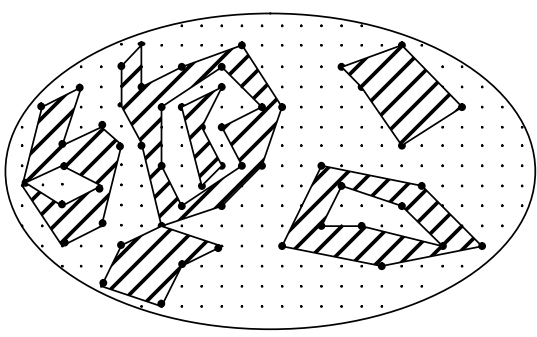

a REGIONS value

Figure 19

It should be obvious now that these data types have very nice closure properties. For example, the set operations are defined as follows. Let $P_{1}$ and $P_{2}$ be two POINTS values, $L_{1}$ and $L_{2}$ two LINES values, and $R_{1}, R_{2}$ two REGIONS values. Then

$$
\begin{aligned}
& \text { union }\left(P_{1}, P_{2}\right):=P_{1} \cup P_{2} \\
& \text { union }\left(L_{1}, L_{2}\right):=\operatorname{blocks}\left(S\left(L_{1}\right) \cup S\left(L_{2}\right)\right) \\
& \text { union }\left(R_{1}, R_{2}\right):=\operatorname{faces}\left(\operatorname{units}\left(R_{1}\right) \cup \operatorname{units}\left(R_{2}\right)\right)
\end{aligned}
$$


For intersection and difference the definitions are analogous. The primitives introduced in the previous sections offer a formal basis for the definition of operations of a spatial algebra. For example, one can define what it means for two regions to be adjacent:

$F$ and $G$ are adjacent $\quad: \Leftrightarrow F$ and $G$ are area-disjoint $\wedge \exists f \in F \exists g \in G: f$ and $g$ are adjacent (assuming area-disjoint to be defined already for regions).

\section{Conclusions}

In this paper we have offered geometric realms as a concept to solve several problems related to spatial data types for database systems. In particular, realms solve the geometric consistency problem as well as problems of numeric robustness and topological correctness. Realm-based structures can be used for the definition of quite general spatial data types and guarantee all the desired closure properties in theory as well as in computational practice. Starting from integer arithmetics, we have developed bottom-up a precise formal framework that makes it easy to define spatial algebras and to implement them correctly. Indeed, such a realm-based algebra is defined in [GüS92].

In closing, let us briefly discuss some open problems and questions that arise with this approach.

Topological correctness. Although it goes a long way, the approach of Greene and Yao does not completely guarantee topological correctness. As is also stated in [GrY86], through the finite representation "... disjoint points and lines may collapse. However, aside from such degeneracies, we do guarantee that topology does not change." There has been a lot of work on numeric robustness and topological correctness for geometric computation (e.g. [OtTU87, GuiSS89, EdM88, NaME90]). We have selected [GrY86] because it fits well with our idea of realms as grid-based planar graphs underlying spatial data types. However, one might try to extend this by adding further integrity constraints (such as our rule that $R$-points must not lie on envelopes) or by techniques from the other approaches (e.g. symbolic reasoning) to avoid the remaining anomalies.

Efficiency. The realm update algorithms of Section 5 have been given in rather abstract terms, one might be concerned, whether they are efficiently implementable. We suggest to represent a realm in a spatial index structure (e.g. [Gut84]) and are currently implementing realms on the basis of LSDTrees [HeSW89]. The lookup operations needed in the algorithms can then be performed efficiently. Such a realm representation can at the same time be used as an index into the database. A separate issue is the efficiency of spatial algebra operations (such as intersecting two regions). This can be done by variations of plane-sweep algorithms such as [NiP82, BeO79]. Indeed, these algorithms are now much simpler and more efficient since they do not need to discover new intersections and do not have to treat special cases. The study of algorithms for realm-based data types might become an interesting field of its own.

Space overhead. By redrawing, many more segments may be created than were present in the original set of intersecting line segments. How many more, is an interesting question that should be studied theoretically as well as in experiments with "real life" data. In any case, we feel one cannot trade correctness for space.

Multiple realms. In this paper we have only discussed the case of a single realm underlying all spatial data (of a certain application area). There are several reasons why one might be interested in several 
realms over the same area. One is to reduce space overhead (by not intersecting spatial values of different realms). Another reason is that there exist interesting SDT operations that are not closed with respect to a realm. An example is the creation of a "buffer area" around a polyline. To accommodate such operations one might dynamically create a realm containing just the "new" spatial values, select a set of SDT values that might interact with these new geometries and create a "small" realm for them, and then use a "merge" operation on realms to compute all intersections correctly.

\section{Appendix: Definition of Geometric Primitives}

As a basis for definition and implementation we only assume that the following arithmetic primitives are available and are error-free with respect to overflow.

$$
\begin{array}{ll}
\mathrm{INT} \times \mathrm{INT} \rightarrow \mathrm{INT} & +,-, * \text {, div, mod } \\
\mathrm{INT} \times \mathrm{INT} \rightarrow \mathrm{BOOL} & =, \neq,<, \leq, \geq,>
\end{array}
$$

To fulfill this requirement in an implementation it is sufficient that the INT data type can represent numbers in the range $\left[-2 n^{3}, 2 n^{3}\right]$ where $n=|N|$ (see below). Either this relationship holds between $N$ needed by the application and a programming language integer type, or one needs to implement a special integer type with these operations.

For an $N$-point $p$ we denote by $p . x$ and $p . y$ its first and second component, respectively. Two $N$-points $p$ and $q$ are equal,

$$
p=q \quad: \Leftrightarrow \quad p \cdot x=q \cdot x \wedge p \cdot y=q \cdot y .
$$

Two $N$-segments $s_{1}=\left(p_{1}, p_{2}\right)$ and $s_{2}=\left(q_{1}, q_{2}\right)$ are equal,

$$
s_{1}=s_{2} \quad: \Leftrightarrow \quad\left(p_{1}=q_{1} \wedge p_{2}=q_{2}\right) \vee\left(p_{1}=q_{2} \wedge p_{2}=q_{1}\right)
$$

Let $s_{1}=\left(p_{1}, p_{2}\right)=\left(\left(x_{11}, y_{11}\right),\left(x_{12}, y_{12}\right)\right)$ and $s_{2}=\left(q_{1}, q_{2}\right)=\left(\left(x_{21}, y_{21}\right),\left(x_{22}, y_{22}\right)\right)$ be two $N$-segments. For the calculation of a possible intersection point of the two $N$-segments we use the following matrix representation where $\lambda, \mu$ are rational numbers (to be represented by pairs of INT values).

$$
\left[\begin{array}{l}
x_{11} \\
y_{11}
\end{array}\right]+\lambda\left(\left[\begin{array}{l}
x_{12} \\
y_{12}
\end{array}\right]-\left[\begin{array}{l}
x_{11} \\
y_{11}
\end{array}\right]\right)=\left[\begin{array}{l}
x_{21} \\
y_{21}
\end{array}\right]+\mu\left(\left[\begin{array}{l}
x_{22} \\
y_{22}
\end{array}\right]-\left[\begin{array}{l}
x_{21} \\
y_{21}
\end{array}\right]\right)
$$

This leads to the following inhomogeneous linear equation system in two variables:

$$
\begin{aligned}
& x_{11}-x_{21}=-\lambda\left(x_{12}-x_{11}\right)+\mu\left(x_{22}-x_{21}\right) \\
& y_{11}-y_{21}=-\lambda\left(y_{12}-y_{11}\right)+\mu\left(y_{22}-y_{21}\right)
\end{aligned}
$$

Let $a_{11}:=x_{11}-x_{12}, a_{12}:=x_{22}-x_{21}, b_{1}:=x_{11}-x_{21}, a_{21}:=y_{11}-y_{12}, a_{22}:=y_{22}-y_{21}$, and $b_{2}:=y_{11}-$ $y_{21}$. Then

$$
\begin{aligned}
& a_{11} \lambda+a_{12} \mu=b_{1} \\
& a_{21} \lambda+a_{22} \mu=b_{2}
\end{aligned} \quad \Rightarrow \quad \begin{aligned}
& \lambda\left(a_{11} a_{22}-a_{12} a_{21}\right)=b_{1} a_{22}-b_{2} a_{12} \\
& \mu\left(a_{11} a_{22}-a_{12} a_{21}\right)=b_{2} a_{11}-b_{1} a_{21}
\end{aligned}
$$

With $D:=a_{11} a_{22}-a_{12} a_{21}, D_{1}:=b_{1} a_{22}-b_{2} a_{12}, D_{2}:=b_{2} a_{11}-b_{1} a_{21}$, and $D \neq 0$ we get

$$
\lambda=\frac{D_{1}}{D}, \mu=\frac{D_{2}}{D} \text {. }
$$


Two $N$-segments intersect if $D \neq 0$ and $0<\lambda<1$ and $0<\mu<1$. Note that the situation where an end point of one segment lies on the other segment is excluded. In particular no two end points are equal. Two $N$-segments are parallel if $D=0$.

For an $N$-segment $s=\left(\left(x_{1}, y_{1}\right),\left(x_{2}, y_{2}\right)\right), x-\operatorname{ext}(s):=\left\{\min \left(x_{1}, x_{2}\right), \ldots, \max \left(x_{1}, x_{2}\right)\right\} \subseteq N$ and $y$-ext $(s):=$ $\left\{\min \left(y_{1}, y_{2}\right), \ldots, \max \left(y_{1}, y_{2}\right)\right\} \subseteq N$ denote the $x$ - and $y$-intervals of its bounding box. The resulting intervals are called $N$-intervals. Two $N$-intervals $I_{1}$ and $I_{2}$ overlap if $\operatorname{card}\left(I_{1} \cap I_{2}\right)>1$. They are disjoint if $I_{1} \cap I_{2}=\varnothing$. Two $N$-segments $s_{1}, s_{2}$ overlap if

(i) $\quad D=0$

(ii) $D_{1}=D_{2}=0$

(iii) $x-\operatorname{ext}\left(s_{1}\right)$ and $x-\operatorname{ext}\left(s_{2}\right)$ overlap $\vee y-\operatorname{ext}\left(s_{1}\right)$ and $y-\operatorname{ext}\left(s_{2}\right)$ overlap.

If condition (iii) does not hold and the $x$ - and $y$-intervals of $s_{1}$ and $s_{2}$ are disjoint, the two $N$-segments are called aligned. Two $N$-segments $s_{1}=\left(p_{1}, p_{2}\right)$ and $s_{2}=\left(q_{1}, q_{2}\right)$ meet if they have exactly one end point in common. Two $N$-segments are disjoint if they are neither equal nor meet nor intersect. If two $N$-segments $s_{1}=\left(p_{1}, p_{2}\right)=\left(\left(x_{11}, y_{11}\right),\left(x_{12}, y_{12}\right)\right)$ and $s_{2}=\left(q_{1}, q_{2}\right)$ intersect, then intersection $\left(s_{1}, s_{2}\right)$ is the $N$-point $(\bar{x}, \bar{y}):=\left(\right.$ round_to_N $\left(x_{0}\right)$, round_to_N $\left.N\left(y_{0}\right)\right)$ where

(i) $x_{0}=x_{11}+\lambda\left(x_{12}-x_{11}\right)$

$y_{0}=y_{11}+\lambda\left(y_{12}-y_{11}\right)$

$x_{0}$ and $y_{0}$ are two rational numbers resulting from solving the two equations in exact rational arithmetic (to be implemented through the INT primitives alone). $\lambda$ is chosen as mentioned in (EQ 1).

(ii) the function round_to_ $N$ rounds a rational number to the "nearest" number in $N$.

For the function round_to_ $N$ we give a simple algorithm to show that integer arithmetic is sufficient to calculate the "nearest" number in $N$ from a rational number $c=\frac{a}{b}$ :

function round_to_N $(a, b:$ integer $):$ integer;

$\operatorname{var} z:$ integer;

begin

if $a \geq b$ then $z:=a \operatorname{div} b ; a:=a \bmod b$ else $z:=0$ end; (* now $a<b$ so that $0<a / b<1$ hold *)

if $a=0$ then return $z$ end;

if $2 * a \leq b$ then return $z$ else return $z+1$ end

end $r o u n d \_t o \_N$;

Let $s=\left(p_{1}, p_{2}\right)=\left(\left(x_{1}, y_{1}\right),\left(x_{2}, y_{2}\right)\right)$ be an $N$-segment and let $p=(x, y)$ be an $N$-point. $p$ lies on $s$, for short: $p$ on $s$, if

(i) $\left(x_{2}-x_{1}\right)\left(y-y_{1}\right)+\left(x-x_{1}\right)\left(y_{1}-y_{2}\right)=0$

(ii) $x \in x-\operatorname{ext}(s) \vee y \in y-\operatorname{ext}(s)$

An $N$-point $p$ lies within an $N$-segment $s$, for short: $p$ in $s$, if additionally to (i) and (ii) holds

(iii) $x \notin\left\{x_{1}, x_{2}\right\} \vee y \notin\left\{y_{1}, y_{2}\right\}$.

One can observe that the largest numbers occur in the equations (EQ 2) 
$x_{0}=\frac{x_{11} D+D_{1}\left(x_{12}-x_{11}\right)}{D}, y_{0}=\frac{y_{11} D+D_{1}\left(y_{12}-y_{11}\right)}{D}$

which leads to the requirement that numbers up to $\left|2 n^{3}\right|$ should be representable.

\section{References}

[AbB88] Abiteboul, S., and C. Beeri, On the Power of Languages for the Manipulation of Complex Objects. Technical Report 846, INRIA (Paris), 1988.

[BeO79] Bentley, J.L., and T. Ottmann, Algorithms for Reporting and Counting Geometric Intersections. IEEE Trans. on Computers C-28 (1979), 643-647.

[EdM88] Edelsbrunner, H., and E.P. Mücke, Simulation of Simplicity. Proc. ACM Symposium on Computational Geometry (Urbana-Champaign, Illinois), 1988.

[EgFJ89] Egenhofer, M.J., A. Frank, and J.P. Jackson, A Topological Data Model for Spatial Databases. Proc. SSD 89 (Santa Barbara, California), 1989, 271-286.

[GaNT91] Gargano, M., E. Nardelli, and M. Talamo, Abstract Data Types for the Logical Modeling of Complex Data. Information Systems 16, 5 (1991).

[GrY86] Greene, D., and F. Yao, Finite-Resolution Computational Geometry. Proc. 27th IEEE Symp. on Foundations of Computer Science, 1986, 143-152.

[GuiSS89] Guibas, L., D. Salesin, and J. Stolfi, Epsilon-geometry: Building Robust Algorithms from Imprecise Computations. Proc. SIAM Conf. on Geometric Design (Tempe, Arizona), 1989.

[Gut84] Guttman, A., R-Trees: A Dynamic Index Structure for Spatial Searching. Proc. ACM SIGMOD Conf. 1984, 47-57.

[Gü88a] Güting, R.H., Geo-Relational Algebra: A Model and Query Language for Geometric Database Systems. Proc. of the Intl. Conf. on Extending Database Technology (Venice, Italy), 1988, 506-527.

[Gü88b] Güting, R.H., Modeling Non-Standard Database Systems by Many-Sorted Algebras. Fachbereich Informatik, Universität Dortmund, Report 255, 1988.

[Gü89] Güting, R.H., Gral: An Extensible Relational Database System for Geometric Applications. Proc. of the 15th Intl. Conf. on Very Large Databases (Amsterdam, The Netherlands), 1989, 33-44.

[GüS92] Güting, R.H., and M. Schneider, Realm-Based Spatial Data Types. FernUniversität Hagen, Manuscript in preparation, 1992.

[HeSW89] Henrich, A., H.-W. Six, and P. Widmayer, The LSD Tree: Spatial Access to Multidimensional Point- and Non-Point-Objects. Proc. of the 15th Intl. Conf. on Very Large Data Bases (Amsterdam, The Netherlands), 45-53.

[JoC88] Joseph, T., and A. Cardenas, PICQUERY: A High Level Query Language for Pictorial Database Management. IEEE Trans. on Software Engineering 14 (1988), 630-638.

[LiN87] Lipeck, U., and K. Neumann, Modelling and Manipulating Objects in Geoscientific Databases. Proc. 5th Intl. Conf on the Entity-Relationship Approach (Dijon, France, 1986), 1987, 67-86.

[NaME90] Nagy, G., M. Mukherjee, and D.W. Embley, Making Do with Finite Numerical Precision in Spatial Data Structures. Proc. 4th Intl. Symp. on Spatial Data Handling (Zürich, Switzerland), 1990, 55-65.

[NiP82] Nievergelt, J., and F.P. Preparata, Plane-Sweep Algorithms for Intersecting Geometric Figures. Communications of the ACM 25 (1982), 739-747.

[OrM88] Orenstein, J., and F. Manola, PROBE Spatial Data Modeling and Query Processing in an Image Database Application. IEEE Trans. on Software Engineering 14 (1988), 611-629.

[OtTU87] Ottmann, T., G. Thiemt, and C. Ullrich, Numerical Stability of Geometric Algorithms. Proc. 3rd ACM Symp. on Computational Geometry, 1987, 119-125.

[RoFS88] Rossopoulos, N., C. Faloutsos, and T. Sellis, An Efficient Pictorial Database System for PSQL. IEEE Trans. on Software Engineering 14 (1988), 639-650.

[ScV89] Scholl, M., and A. Voisard, Thematic Map Modeling. Proc. SSD 89, (Santa Barbara, California), 1989, 167-190. 
[SvH91] Svensson, P., and Z. Huang, Geo-SAL: A Query Language for Spatial Data Analysis. Proc. SSD 91 (Zürich, Switzerland), 1991, 119-140.

[Vo92] Voisard, A., Bases de données géographiques: du modèle de données à l'interface utilisateur. Ph.D. Thesis, University of Paris-Sud (Centre d'Orsay), 1992. 